\title{
Construction-Centric Building Information Modelling to Facilitate Building Panel Prefabrication
}

\author{
Hexu LIU ${ }^{1}$, Gurjeet SINGH ${ }^{1}$, Youyi ZHANG ${ }^{1}$, Ming LU ${ }^{1} *$ and Mohamed AL-HUSSEIN ${ }^{1}$ \\ 1 Department of Civil and Environmental Engineering, University of Alberta, Canada \\ *Corresponding author's e-mail: mlu6@ualberta.ca
}

\begin{abstract}
Building information modelling (BIM) is an information technology that has the ability to change the Architecture, Engineering, and Construction (AEC) industry in terms of enhanced communication and productivity. With the rise of BIM, panelized/off-site construction is gaining momentum within the home building industry. However, BIM has not yet been leveraged to its full capability with regard to construction prefabrication, due in part to the fact that BIM models are only roughly designed by architects and engineers. Furthermore, some construction-centric information, such as drywall and sheathing layouts, which require substantial manual modelling efforts, is not presented explicitly in the BIM model. In this regard, this research exploits a BIMbased automatic approach for designing and modelling drywall and sheathing layouts in order to facilitate building panel production in the prefabrication plant. The prototype system is built on the basis of Autodesk Revit through the use of Application Programming Interface (API). This system, taking architectural BIM information as the input, generates a detailed constructioncentric BIM model in accordance with construction specifications, and further enables users to obtain shop drawings and a thorough quantity take-off and cut list in order to manage the plant production. In addition, the prototype system is capable of optimizing drywall and sheathing layout design with the objective of minimizing material waste and joint length. A case study of a wood-framed residential building is adopted to demonstrate the developed prototype system.
\end{abstract}

\section{KEYWORDS}

Construction-centric BIM, Panelized construction, Sheathing and drywall optimization.

\section{INTRODUCTION}

Since the time of the industrial revolution efforts to improve efficiency have served as a catalyst for innovation, with engineers inventing new processes or improving existing ones by means of new managerial approaches. In this context, the quest for efficiency in the field of building construction has led to the emergence of modularization, which in turn has bolstered the expansion of off-site construction methods. Prefabrication construction introduces improved quality and reduced cycle time, and allows for higher safety standards to be implemented since work is performed within a controlled environment. In the meantime, it also introduces new challenges which necessitate the implementation of innovative technologies such as building information modelling (BIM). BIM, as a digital representation of physical and functional characteristics of a facility, can assist owners and AEC (Architecture, Engineering, and Construction) organizations in efficiently planning, designing, constructing, and managing 
building and infrastructure projects. Owners, architects, contractors, engineers, and fabricators are eager to implement BIM in their organizations and include it as a permanent part of their overall project delivery mechanism. As such, BIM has been increasingly employed in the construction industry. McGraw-Hill Construction (MHC 2011) has reported that the use of BIMdriven prefabrication on more than one quarter of their projects is expected to increase from $37 \%$ to $73 \%$ among practitioners.

BIM, however, currently still lacks sophistication on the construction side, partially due to the extensive manual efforts required in construction-centric BIM. BIM model, in general, is categorized into five level of developments (LOD), ranging from LOD 100 to LOD 500 (ASBO 2013). BIM model cannot satisfy the requirements of contractors and fabricators when it has been only roughly designed by architects and engineers (i.e., to LOD 300 or less), since only BIM model at LOD 350 or more is capable of showing detailed subcomponents (e.g., blocking, king studs, and wall bracing, etc.) of building components (Webster, 2014). Construction-centric BIM in this study refers to a BIM model at a level of development of 350 or more, and it is usually not subjects of interest to architects and engineers because of the substantial manual efforts. Monteiro et al. (2013) concluded that the modeling time in ArchiCAD for structural elements approximately doubles when their formwork is modeled. Similar to formwork, precise sheathing and drywall layout information is not represented explicitly in BIM models developed by architects and structural engineers, and significant efforts are required from construction practitioners to leverage BIM models with this information according to construction specifications and design principles.

Improper planning and modelling for boarding of residential houses (sheathing and drywall layout) is widely acknowledged as a major cause of material waste (Mogollon 2009). Despite the fact that parametric modelling can be utilized to define parametric objects for modelling sheathing and drywall sheets, placing individual boarding sheets at the correct design location is time-consuming and error-prone. Additionally, parametric modelling, as a modelling technology, cannot provide stakeholders with optimized design solutions which reduce material waste and construction cost. This research thus proposes a BIM-based automated design and modelling approach for drywall and sheathing layouts in order to facilitate building panel production in the prefabrication plant. This automated approach, taking architectural BIM information as the input, generates a detailed construction-centric BIM model in accordance with construction specifications. It further enables users to obtain shop drawings and a thorough quantity take-off and cut list automatically in order to manage plant production. In addition, a cutting stock solver is integrated into the prototype system in order to optimize drywall and sheathing layout design with the objective of minimizing material waste and joint length. The developed prototype system is built on the basis of Autodesk Revit through the Application Programming Interface (API).

The design principles pertaining to boarding of residential buildings are explained in this paper. Subsequently, the BIM-based methodology of detailed construction information modelling for sheathing and drywall layout is illustrated in detail. Along with this, optimization of boarding design is presented. A case study of a wood-framed residential building is also presented to demonstrate the effectiveness of the methodology and the prototype system. Conclusions are summarized and limitations of the present research are identified with respect to future research. 


\section{DESIGN PRINCIPLES FOR BOARDING OF RESIDENTIAL BUILDINGS}

Sheathing and Drywall boarding requires cutting drywall sheets to proper sizes and then screwing (or gluing) them to the wood (or metal) studs. Though a very simple and straightforward exercise, it is crucial to follow certain design principles when laying out sheathing and drywall sheets. Sheets of drywall are available in rectangular shapes with different sizes and varying thicknesses. The most commonly used drywall sizes in residential construction are 4'x 8', 4'x9', 4'x10', 4'x12', 5'x8', 5'x9', 5'x10', and 5'x12'. The design principles aim to minimize butt joints (between butt edges) and wasted drywall, as well as reduce cracks at seams. These requirements make the pattern of laying the sheets of drywall on the studs very crucial.

Generally, beveled factory edges (long edge of a drywall sheet) should adjoin other factory edges, and butt edges (short side of a drywall sheet) should adjoin other butt edges. When two factory edges meet, a recess for filling in the joints is created, which makes taping and feathering seams much easier. Provided that a drywall butt edge is aligned next to a factory edge, it causes uneven surfaces that make taping and finishing much more difficult. At the same time, butt joints should be minimized whenever possible as the area around the joint does not become flush with other areas of the drywall sheet after finishing. Sheets of drywall should always be laid out perpendicular to the direction of wood studs where the sheets splice on the stud. If the drywall is placed parallel to the studs, the factory (long) edges might not align directly in the middle of the stud when studs are twisted or crooked. Furthermore, the drywall may not have proper backing if the studs have these imperfections. When laid perpendicular to the studs, the resulting drywall structure is stronger and has greater resistance to cracking at the seams due to an increase in holding power across the wall as more studs are connecting together. Also, in such a layout the seams are in the middle of the wall, making it easier to complete the tasks of taping and finishing. As such, placing sheathing and drywall sheets on walls horizontally is a common practice in the housing industry, and boarding cutting pattern is formulated as a one-dimensional cutting stock problem in this study. In addition to this, drywall butt joints should always be staggered. This leads to increased overall strength of the wall. Since butt joints are prone to cracking, staggered layout limits them to no more than 4' in length. Drywall can be hung either from left-to-right or from right-to-left along a wall. The sheets on a second wall will overlap the sheets on the first wall, creating a very tight corner between the first and second wall. The areas around openings (doors and windows) are high-stress areas (with seams prone to cracking due to opening and closing of doors), which means joints should be avoided close to such locations.

\section{METHODOLOGY}

This research adopts an automated approach to construction-centric building information modelling with a focus on sheathing and drywall in residential building projects, thereby facilitating building panel production in prefabrication shops. An automated design and modelling prototype system is developed on the basis of the Autodesk Revit platform using an application programming interface in $\mathrm{C \#} \mathrm{language.} \mathrm{The} \mathrm{reasons} \mathrm{for} \mathrm{selecting} \mathrm{Autodesk} \mathrm{Revit} \mathrm{as}$ the BIM platform in this study are as follows: (1) Revit has a strong presence in North America, which allows the proposed planning system to be easily adopted into the current industry; (2) Revit is a powerful modelling tool, which gives end-users modelling flexibility by means of its built-in functions such as Family Editor; (3) Revit supports API at the programming level; and (4) Revit supports Industry Foundation Classes (IFC), which addresses problems of interoperability. Figure 1 presents the architecture of the proposed Revit-based automatic design 
and modelling system. The inputs for the proposed system include: (1) building design for the project, such as a BIM model containing architectural and structural information; (2) material sizes, which indicate the standard sizes of drywall and sheathing boards in market; and (3) boarding design principles, which allow users to select boarding design rules commonly adopted in the residential building industry. The core of this prototype system, as shown in the middle of Figure 1, consists of four components: (1) BIM model parser, which extracts building semantic and geometrical information for the downstream design analysis; (2) rule-driven drywall and sheathing boarding design algorithm, which is used to design the drywall and sheathing layout in accordance with boarding design rules; (3) cutting stock optimizer (Montibelli A, 2014), which is employed to optimize the material sheet cutting with the objective of minimizing material waste and material cost; and (4) drywall and sheathing layout modeller, which takes the optimized design parameter as input and shows layout design in the BIM model. The four components are encoded into Autodesk Revit as add-on through the application programming interface (API) in C\# language. It is worth noting that the boarding design algorithm generates various feasible design scenarios and feeds them into the cutting stock optimizer. Afterward, the cutting stock optimizer determines cutting patterns by solving for a one-dimensional cutting stock problem, and calculates the waste ratio and material cost of each scenario. In the end, an optimized design scenario with shop drawings and a cutting list is produced in order to assist production managers in planning and managing the panel production. The output of this system also comprises a construction-centric BIM model which allows construction practitioners to visualize the boarding design in 3D. Detailed illustrations of the automatic approach are discussed below.

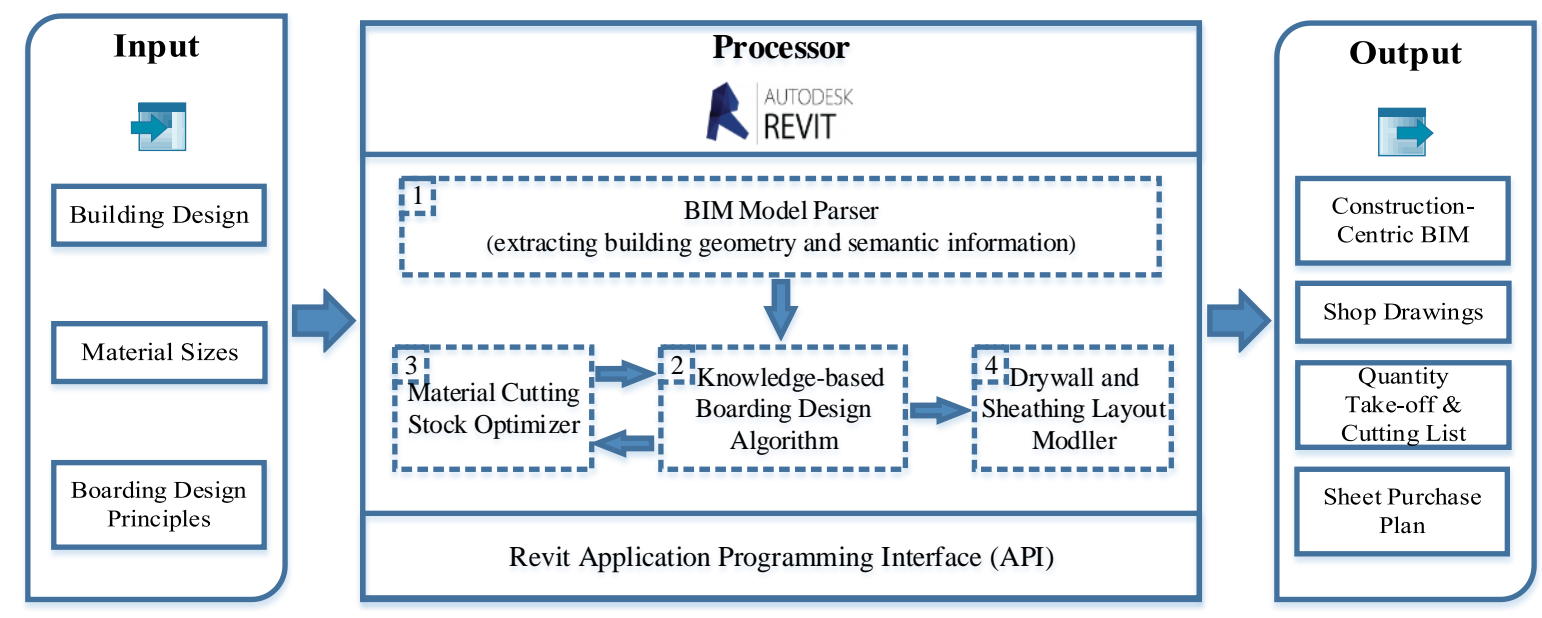

Figure 1. Architecture of automatic design and modelling system

\section{SEMANTIC-DRIVEN BUILDING INFORMATION EXTRACTION}

Generally speaking, building information in the BIM model can be categorized into three groups including geometry, topology, and semantic information. Geometric information refers to dimensions and locations of building components, while topological information is to elaborate their spatial relationships. Semantic information refers to additional attributes or properties describing building components, such as host information and functional information (e.g., interior, exterior, and structural, etc.). The first two types of information can be held by and derived from traditional 3D CAD models, while semantic information is embedded in the BIM 
model as property. In theory, sheathing and drywall modelling necessitates geometrical and topological information. As such, a few 3D CAD-based attempts to model sheathing and drywall design have been successfully carried out in previous research (e.g., Mogollon 2009). However, semantic information boosts the efficiency of information extraction and enhances the traditional 3D CAD-based modelling approach by eliminating certain geometrical analyses. For example, "Host by" between openings/studs and walls can be easily recognized by the semantic host property of building elements, rather than through complex pure geometrical analysis. Additionally, semantic material information of wall layers is beneficial for identifying and extracting geometrical information of specific wall layers. Hence, a BIM model parser is developed in this study in order to extract semantic and geometrical information, thereby facilitating sheathing/drywall boarding design and modelling.

Figure 2 illustrates the methodological flowchart of the developed BIM model parser. To begin, the developed BIM model parser retrieves all building elements relevant to sheathing and drywall design and modelling, such as wall, window, door, and stud. Subsequently, their semantic information such as host information (e.g., panel name) and wall layer (e.g., material) is retrieved through Revit API, and is used to detect relationships between openings/studs and walls. On this basis, geometrical information such as location of studs and windows is then extracted. It should be noted that wall elements in building design usually consist of various layers, depending on the architectural design. Individual boarding sheets are designated within the sheathing and drywall layers in the wall. To achieve this, geometrical information for an individual sheathing and drywall layer is extracted using the Revit API functions of element.get_Geometry(), solid.Faces(),face.EdgeLoops(), and Curve.GetEndPoint() in the BIM model parser. Figure 3 presents a sample sketch of a wall panel and its associated sheathing and drywall layers and openings. Geometrical information (e.g., vertices of drywall and sheathing layers) extracted by the BIM model parser is represented by the red dots in Figure 3 . This information, along with formalized design principles, is used to design boarding in the following section.

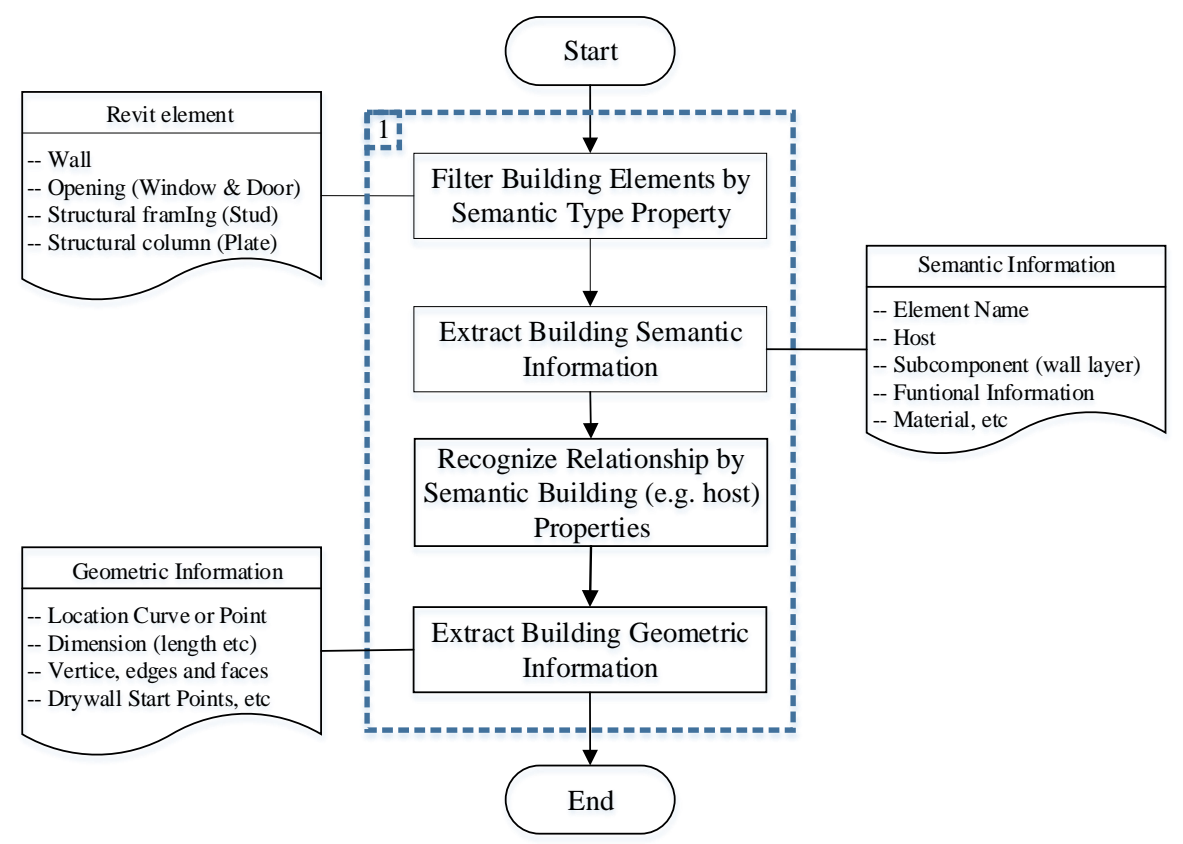

Figure 2. Methodological flowchart of the developed BIM model parser 


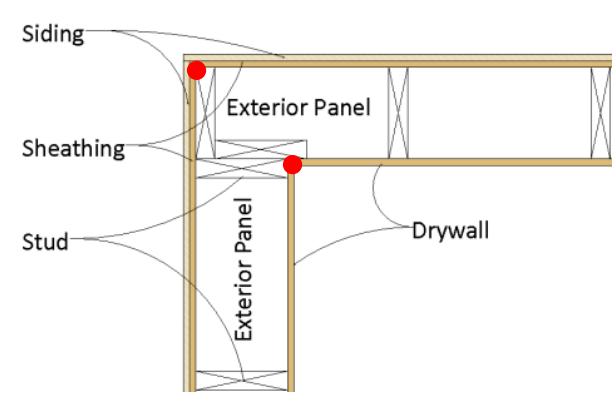

(a) L - Connection

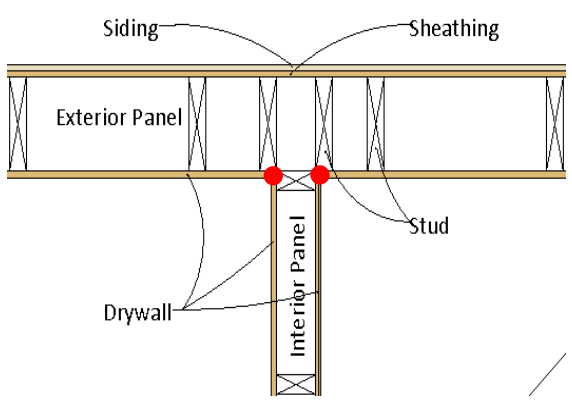

(b) T - Connection

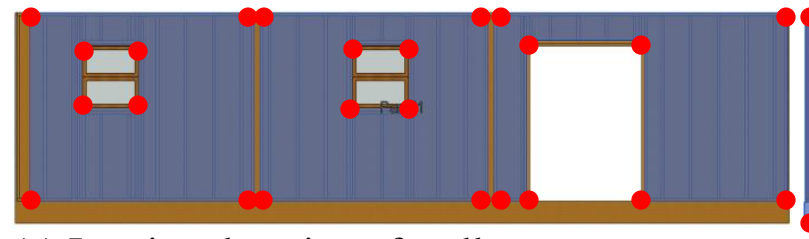

(c) Interior elevation of wall

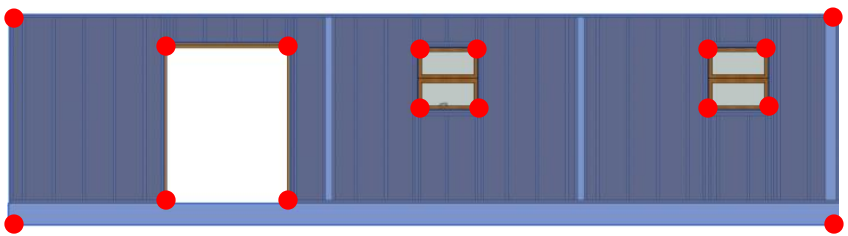

(d) Exterior elevation of wall

Figure 3. Sketches of wall layers

\section{KNOWLEDGE-BASED OPTIMIZED BOARDING DESIGN}

Once semantic and geometrical information is retrieved from the BIM model, a rule-based boarding design algorithm is launched. The methodological flowchart of optimizing boarding design is presented in Figure 4. In general, sheathing and drywall design is an iterative process. The design algorithm begins by selecting one standard boarding size (e.g., 4' $\times 8^{\prime}$ ) and identifies the start point of the sheathing and drywall wall layer. Subsequently, one sheathingldrywall board of the selected size is placed horizontally at the identified start point, and the end point of the sheathingldrywall board is then calculated. Afterward, this end point is checked against the design principles in order to ensure that formalized design principles, such as Lay drywall edge on stud, Stagger drywall edge, and Avoid edge around opening seam, are satisfied. In case of any incompliance, the sheathing/drywall board is cut down to adjust its end point and a new end point satisfying all design rules is re-calculated by the algorithm. This end point then serves as a new start point at which to place the next sheathing/drywall board. The processes do not terminate until all boarding sheets are placed on walls. Upon completion of configuring boarding design for all wall panels, the cutting stock solver is then triggered to formulate an optimized cutting pattern, which minimizes cutting waste and material cost for this design scenario. Three algorithms, Greedy First Fit, Greedy Best Fit, and Greedy Next Fit, are executed sequentially in the cutting stock solver. For a given design scenario, the optimized material cost and cutting waste can be obtained from the cutting stock solver. After saving this design scenario, the next iteration is then triggered and another standard boarding size (e.g., 4' $\times 10^{\prime}$ ) is selected by the algorithm to formulate a new design. The algorithm does not terminate until all available standard boarding sizes are iterated to design the boarding layout. Finally, the design with the least amount of material cost or cutting waste is identified by the prototype system, and then modelled automatically in the BIM model by the sheathing and drywall layout modeler (shown in Figure 1) in order to generate shop drawings and obtain a construction-centric BIM model. The successful design is also used to formulate the Microsoft Excel-based boarding sheet purchase plan and cutting list, which is automatically generated by the prototype system. 


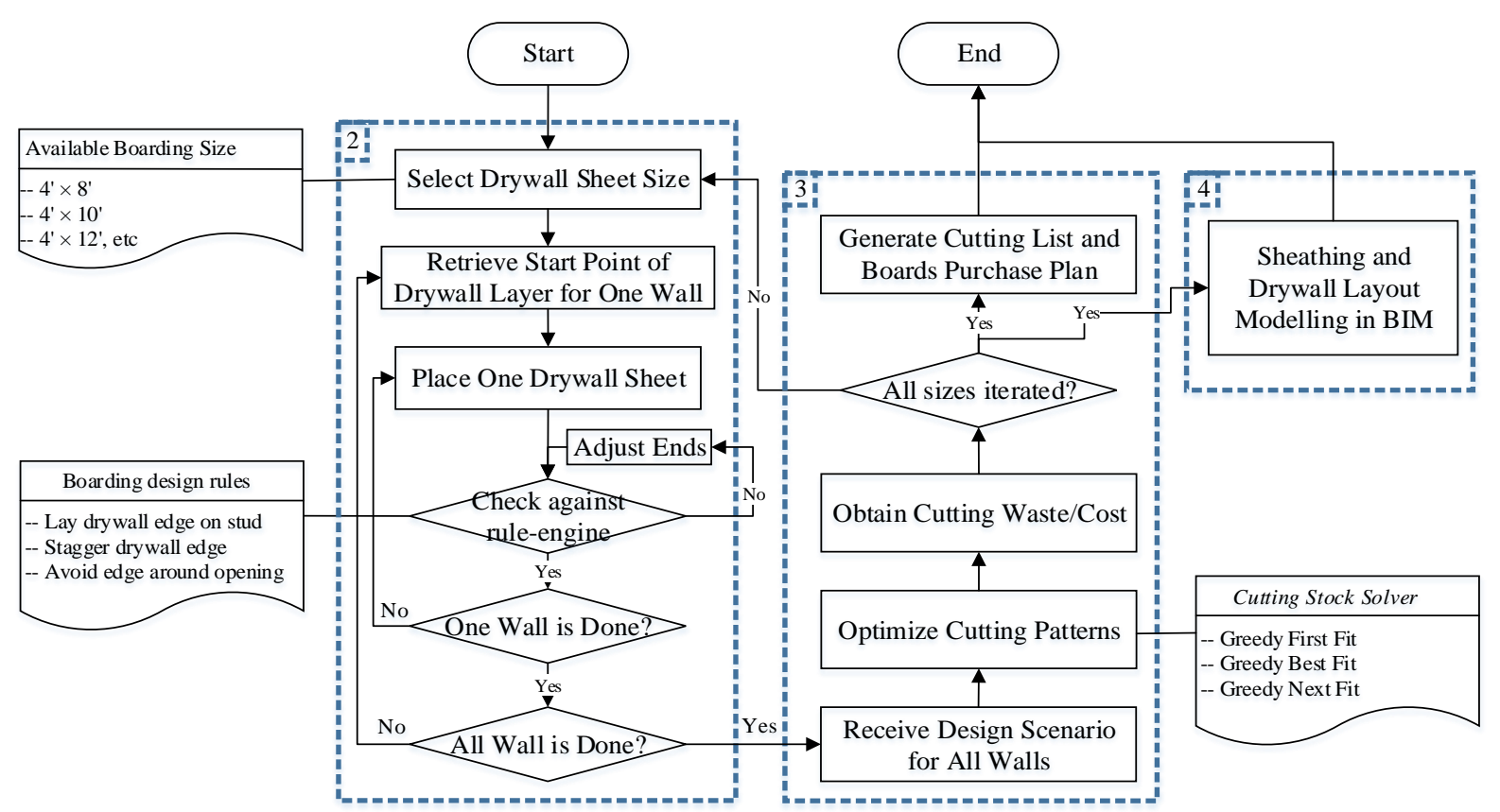

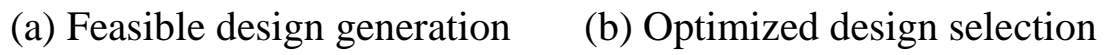

Figure 4. Methodological flowchart of optimizing boarding design

\section{CASE STUDY}

The developed prototype system is tested in a wood-framed residential building as shown in Figure 5. The building model is first built in Autodesk Revit 2015. Due to the fact that Autodesk Revit, as a general BIM modelling tool, does not provide efficient functionalities for detailed construction framing, a suite of commercial Revit add-ons, Metal Wood Framer (MWF) (StrucS-

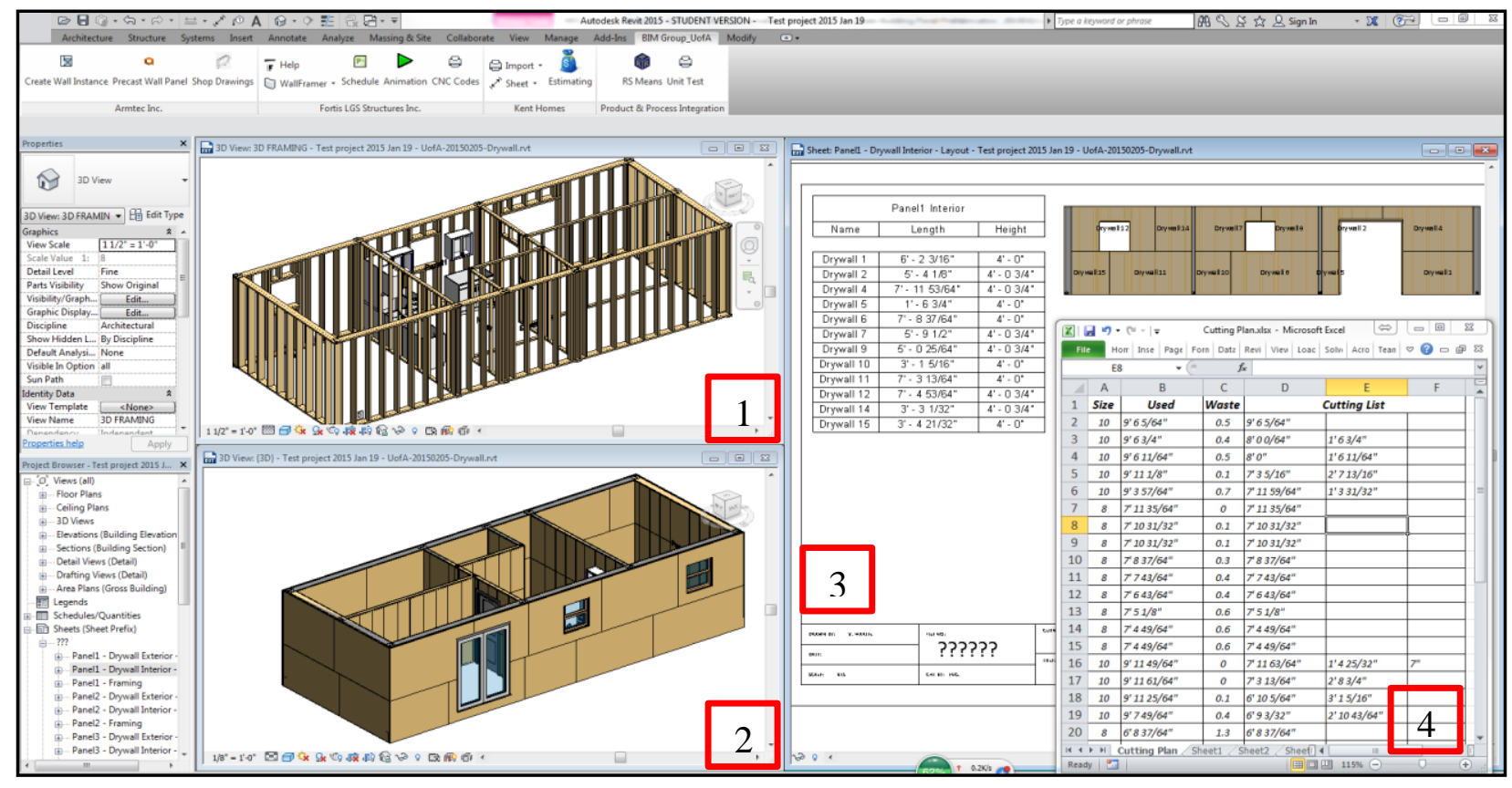

Figure 5. Case study of wood-framed residential building 
oft Solutions 2015), is employed to frame wall panels. Then, the developed prototype system is launched inside Autodesk Revit to design the boarding layout. Construction practitioners need to provide available standard board size and corresponding unit cost information through the graphic user interface to this prototype system, thereby enabling design optimization. The system outputs, including construction-centric BIM, shop drawings with quantity take-off, and cutting list and boarding sheet purchase plan (Figure 5), are generated automatically. Based on this information, construction practitioners can plan and manage the prefabrication.

\section{CONCLUSION}

This research seeks an automatic approach to construction-centric building information modelling with the focus on boarding layout design for residential houses. An Autodesk Revitbased automated design and modelling prototype system has been developed through Revit API. This prototype system incorporates common boarding design principles and cutting stock optimization in order to provide optimized boarding design to stakeholders. Furthermore, a semantic-driven BIM model parser is developed as the basis of this study to enable BIM-based automated design. Compared with traditional 3D CAD technology, BIM provides more effective information extraction, capitalizing on semantic information in the BIM model. The prototype system is tested using a wood-framed residential building project, and is demonstrated to be a helpful tool in assisting stakeholders to plan and manage the prefabrication of the project. In the future, a combination algorithm can be incorporated into this prototype in order to optimize boarding design more efficiently.

\section{ACKNOWLEDGEMENTS}

We gratefully acknowledge the financial support of the Natural Sciences and Engineering Research Council of Canada (NSERC), as well as the assistance from Star Prebuilt Homes Ltd, Edmonton, Canada.

\section{REFERENCES}

Association of School Business Officials (2013). "BIM Resource Guide: A Guide for Implementing Building Information Modeling in State of Maryland and Washington DC Public School Construction Projects."

McGraw-Hill Construction (2011). "Prefabrication and modularization. Increasing productivity in the construction industry."

Mogollon, J. (2009). "Automation of Design and Drafting for Wood Frame Structures and Construction Waste Minimization" Ph.D. thesis, University of Alberta.

Monteiro, A. and Martins, J. (2013) "A survey on modeling guidelines for quantity takeofforiented BIM-based design." Automation in Construction, 35, 238-253.

Montibelli, A. (2014). "Application for solving Bin Packing and Cutting Stock problem." Available from http://www.codeproject.com/Articles/706136/Csharp-Bin-Packing-CuttingStock-Solver

StrucSoft Solutions Ltd. (2015). Metal Wood Framer (MWF) add-on. Available at: http://www.strucsoftsolutions.com/

Webster, D. (2014). "What BIM can be: LOD 300: Optimizing your model for construction documentation." Available from http://www.mastergraphics.com/wordpress/2013/what-bim-canbe-lod-300-optimizing-your-model-for-construction-documentation/ 